\title{
The predicted impact of the NHS White Paper on the use and funding of a specialist service for personality disordered patients: a survey of clinicians' views
}

\author{
Bridget Dolan, Research Fellow in Forensic Psychiatry; and KIngsLey NorTON, \\ Consultant Psychotherapist and Honorary Senior Lecturer, Henderson Hospital, \\ 2 Homeland Drive, Sutton, Surrey SM2 5LT, and Section of Forensic Psychiatry, \\ St George's Hospital Medical School, Tooting, London SW17 0RE
}

In an earlier report (Dolan \& Norton, 1990) we commented upon the potential vulnerability of specialist psychiatric units following the implementation of Working for Patients and the NHS Bill. Specialist units provide a valuable resource, not only in terms of treatment, but as sources of practical training, education, development of new therapies and of research. Many such units previously enjoyed financial protection at the regional health authority level. Downloading financial responsibilities to DHAs or NHS trusts will complicate the issue of funding since contracts will now need to be entered with each relevant DHA or NHS trust. This more cumbersome system is inevitably more costly for a specialist unit to operate. Users of such units (customers and consumers) will find referral problematic since funding must be identified in advance. Difficulty in negotiating the new, more complicated contracting system, may lead to a decline in specialist referrals. This will in turn lead to questioning of how necessary such 'regional' or (as in the case of Henderson Hospital) 'national' specialities really are. Managers may tend to argue that referral patterns will reflect (eventually) the true needs for treatment of particular client groups.

Contracts and billing processes for non-specialist psychiatric treatment of catchment area populations of a given district, which can be made upon the basis of the last year's usage, are relatively straightforward. However, for specialist services (particularly specialist in-patient units) the situation is different. Numbers referred per district are much smaller and identification of funding will need to be made upon a 'cost per case' basis. A given DHA/NHS trust may not need to use the specialist service every year and so will not have a regular budget for it. 'Cost per case' may mean that the clinician has to argue the case for funding each time. In the instance of the Henderson Hospital the clients, people with moderate to severe personality disorders, already represent a marginalised sector of society. Such patients are extremely demanding upon health care professionals' time and resources and often an attitude of therapeutic pessi- mism towards patients with personality disorders has been adopted. The nihilistic attitudes towards such patients has led to them being described as "the patients psychiatrists dislike" (Lewis \& Appleby, 1988). How attractive are such individuals when much of the budget will already have been allocated to other client groups, who often will be seen to suffer from 'more deserving' ailments (in the political sense at least).

Of course it has been vehemently argued, by those supporting the free market approach, that if a specialist unit is truly providing a relevant service that its financial viability is assured. It should have no trouble forging contracts, competing and surviving in the 'new' NHS. However, we would argue that unfortunately this assertion may not be true. Decisions of whether to buy treatment from a specialist unit will be made ultimately by managers, considering budget limitations, rather than by psychiatrists, considering clinical need. Such disenfranchisement of clinicians could lead to the loss of many specialist units and their expertise since the disempowered psychiatrist may not wish to, or be able to, put aside adequate time to press for 'cost per case' funding for each individual patient in need of specialist care. Clinicians' attitudes and their energies in pursuing funding for specialist psychiatric treatments will be central to the survival of such resources.

To illustrate some of the above points we report the findings of a recent marketing survey of our 'customers' (i.e. referrers) regarding Henderson Hospital's therapeutic community approach to treating personality disordered clients.

\section{The study}

Subjects. We surveyed 80 referrers whose clients had been admitted to Henderson over the past four years, regardless of the length of admission of the client. All referrers from whom more than one client had been admitted were included, plus one in five of all other referrers. After three mailings a total of 66 completed responses were obtained, this represents an $82.5 \%$ 
response rate. The majority of referrers, and thus respondents, were psychiatrists $(71.2 \%)$. The remainder comprised: social workers $(10.6 \%)$, general practitioners $(7.6 \%)$ psychologists $(7.6 \%)$, and $3 \%$ who were psychotherapists, probation officers or other health professionals.

Questionnaire. The questionnaire asked opinions upon whether the client had been helped by the admission and the satisfaction with the service offered, specific positive or negative aspects of the service, suggestions for improvements in the service, whether the referrer would, in the future, refer a client for admission, assessment or management advice and finally their anticipation of difficulties funding referrals following the NHS Bill's implementation.*

\section{Questionnaire responses}

The majority of referrers felt that the admission had helped the client, and only $18 \%$ felt that admission had not helped the client, although in several of these cases it was noted that the client had only had a very short admission to Henderson.

Three-quarters of respondents expressed their satisfaction with the service provided by Henderson. Interestingly this included a number of those referrers whose clients had left early. Only seven referrers $(10.6 \%)$ said they were not satisfied with the service provided. When asked which specific aspects they felt were negative, six referrers said they were not satisfied with the discharge procedure, one felt the speed of assessment was too slow, and four did not agree with the model of therapy used at Henderson (specifically two of these felt clients should be given psychotropic drugs, which are never prescribed on the unit).

Particularly positive aspects of the Henderson service were commented upon by 31 respondents $(47 \%)$. These mostly related to the totality of the service provided (23 respondents) and to the group assessment procedure (eight respondents). However, the need for improvements in administration was also noted, in particular greater liaison with referrers was mentioned by $11(16.7 \%)$ people and five $(7.6 \%)$ were not satisfied with the discharge and aftercare procedure.

Almost all respondents (94\%) said they would refer a client for admission to Henderson in the future. However, $19(29 \%)$ said they would only refer a client if the service were free. Again nearly all respondents $(91 \%)$ said they would ask Henderson to assess and advise upon clients in the future but $22(33 \%)$ would only use this service if it were free.

* Further details of the procedure, questionnaire and results are available from Dr Dolan.
The majority of respondents $(82 \%)$ anticipated problems in funding specialist places for personality disordered clients once the NHS bill is implemented. Only five $(7.6 \%)$ felt there would be no problem while the remaining seven $(10.6 \%)$ did not know.

Several respondents commented that they felt it would be impossible to get monies for this group and particularly those professionals in social services felt that personality disordered clients would be unsupported in the future. Encouragingly, 55 (83\%) of respondents said they would be prepared to try to persuade their local Director of Public Health or Hospital Manager to financially support a future referral to Henderson, although many were pessimistic about the outcome of such lobbying.

\section{Comment}

The survey illustrates a number of important points related to both clinical and service issues. Clearly, conclusions from a sample of 66 people must be tentative. Nevertheless, the survey had a response rate of $82 \%$ which could perhaps reflect the importance placed by referrers upon being given an opportunity to express their views concerning provision of psychiatric services following the implementation of the White Paper.

Only seven $(11 \%)$ respondents said they were not satisfied with the service Henderson offered. It is telling that although $74 \%$ of referrers said they were satisfied with our service, several mentioned improvements which could be made. Although pleased to find such wide approval of our service we take seriously the comments made by some respondents regarding improving our liaison with referrers and our discharge procedure. The survey has proved a revealing exercise in enabling us to learn the views of our 'customers' about which aspects of our service warrant specific attention.

Of respondents, $94 \%$ said they would refer a client to Henderson Services in the future. We took this as again indicative of general satisfaction with our service bearing in mind the caveats above. The large majority would approach Henderson for assessment and management advice, again indicating how the expertise of our specialist service as a source of advice and evaluation is regarded by referrers. However, financial constraints apply to asking for this advice by the quarter of respondents who would only want this management advice if it were offered for free; similarly one-third would only request assessments or admission if free.

Of concern, given the need for referral to and advice from Henderson demonstrated above, the vast majority $(82 \%)$ of respondents anticipated problems in securing funding for personality disordered clients once the NHS bill is implemented. Although many respondents stated they would be prepared to try to 
persuade their local budget holder to support referral to Henderson, many additional comments indicate feelings of impotence in achieving this aim.

The pessimism reported in an earlier study regarding psychiatrists' attitudes towards prognosis for personality disorders (Lewis \& Appleby, 1988) was not found in our survey. Three-quarters of referrers who responded to the question felt that admission to Henderson had helped their client with their problems. Perhaps this difference is because our survey dealt only with those professionals who had actually seen and referred a PD client for treatment rather than the hypothetical client in the Lewis \& Appleby survey. Although many short term 'crisis responses' are ineffective as therapeutic interventions, the long term efficacy of in-patient psychotherapy or a therapeutic community approach in managing personality disorders has been demonstrated (Kernberg, 1982; Vaglum et al, 1990; Dolan et al, 1990). In contrast to the 'crisis response' approach, which is demanding of professionals time and effort and is difficult to cost accurately, such interventions may initially seem more expensive, but if effective are cheaper in the longer term.

It seems that for our referrers the pessimism surrounding personality disordered clients is not actually related to their 'treatability' per se but more worryingly it is linked to the feelings that post the NHS Bill such clients will be 'bottom of the pile' in terms of funding. Perhaps "the patients psychiatrists dislike" will fast become "the patients budget managers dislike" as well.

\section{References}

Dolan, B. M. \& Norton, K. (1990) Is there a need to safeguard specialist psychiatric units in the NHS? Henderson Hospital: a case in point. Psychiatric Bulletin, $14,72-76$

, Wilson, J. \& Evans, C. D. H. (1990) Therapeutic community treatment for personality disordered adults: a long term follow up study paper presented at British Psychology Society London Conference.

KERNBERG, O. (1982) Advantages and liabilities of the therapeutic community. In The Individual and the Group (ed. M. Pines). New York: Plenum Press.

LEWIS, G. \& APPLEBY, L. (1988) Personality disorders: the patients psychiatrists dislike. British Journal of Psychiatry, 153, 44-49.

VAglum, P. et al (1990) Treatment response of severe and non-severe personality disorders in a therapeutic community day unit. Journal of Personality Disorders, 4 , 161-172.

\title{
The physical health of old long stay in-patients in one psychiatric hospital
}

\author{
Mary Stewart, Senior Registrar, Andrew Duncan Clinic, Royal Edinburgh Hospital, \\ Edinburgh EH10 5HF
}

The provision of care for patients with chronic mental illness is changing with the gradual closure of mental hospitals and the expectation that this group will be largely cared for in the community. In Scotland the process is only beginning. A recent Scottish survey examined the demographic, social and clinical characteristics of 2605 "old long-stay" in-patients, defined as those in hospital more than six years and under 65 years of age at the time of last admission (McCreadie et al, 1991). Patients in 18 psychiatric hospitals serving $83 \%$ of the Scottish population were studied. Results showed the population was elderly, $70 \%$ being over 60 years of age, and nearly $40 \%$ over 70 years. The aim of the present study was to look at the physical health of the old long-stay population in one hospital, namely Crichton Royal, Dumfries, because of the implications of physical health care needs for the management of this group in the community.

\section{The study}

The survey recorded the age, sex and diagnosis (ICD9, World Health Organization, 1978) of the 168 old long-stay patients from Crichton Royal Hospital, Dumfries who were survivors of McCreadie's study (1991), the present one being carried out five months later. By interviewing senior nurses who had known 